\title{
The ball and stick neuron model accounts both for microscopic and macroscopic power laws
}

\author{
Klas H Pettersen", Henrik Lindén, Tom Tetzlaff, Gaute T Einevoll \\ From Twentieth Annual Computational Neuroscience Meeting: CNS*2011 \\ Stockholm, Sweden. 23-28 July 2011
}

Ever since Hans Berger recorded the first human electroencephalogram (EEG) in 1924 [1] its features have been under extensive study, especially since many of them are directly related to disease and to states of consciousness. In the last decades, the underlying background spectra (the power spectral density, PSD) of the EEG have also attracted great attention. The PSD is often well fit by a $1 / \mathrm{f}^{\alpha}$ power law, with $\alpha$ typically in the range from 1 to $2[2,3]$.

Linking features seen in global recordings, such as the EEG, to features in the underlying local activity, such as single neuron activity, is still a major challenge within the field of neuroscience. Power laws are, however, recorded both at the macroscopic level, e.g., for the EEG or the MEG, and at the level of single neurons. The PSD of the sub-threshold soma potential has been shown to exhibit a $1 / \mathrm{f}^{\alpha}$ power law, but with a larger power $\alpha$ than for the EEG. For the sub-threshold soma potential $\alpha$ is typically ranging from 2 to 3 [4-8]. As for the EEG, this power law seems to be very robust; it has been observed in single neuron recordings across several species and brain regions, and also in cell cultures [4-8].

Here, we present analytical solutions to the ball and stick neuron model with input currents spread homogeneously throughout the dendritic stick. Expressions for the PSD of the soma potential and the PSD of the single neuron contribution to the EEG are derived and shown to follow $1 / f^{\alpha}$ power laws with values of $\alpha$ in agreement with experiments. The scale-free background spectra of the EEG may therefore originate from stochastic processes within the neuronal membrane, rather than relying on complicated network dynamics or self-critical states.

\section{Acknowledgements}

This project was supported by the Research Council of Norway (eVITA [eNEURO], Notur).

Published: 18 July 2011

\section{References}

1. Berger H: Über das Elektrenkephalogramm des Menschen. European Archives of Psychiatry and Clinical Neuroscience 1929, 87:527-570.

2. Buzsáki G: Rythms of the brain. Oxford University Press; 2006.

3. Freeman WJ, Holmes MD, Burke BC, Vanhatalo S: Spatial spectra of scalp EEG and EMG from awake humans. Clin Neurophysiol 2003, 114(6):1053-1068.

4. Diba K, Lester HA, Koch C: Intrinsic noise in cultured hippocampal neurons: experiment and modeling. J Neurosci 2004, 24(43):9723-9733.

5. Rudolph M, Pelletier JG, Paré D, Destexhe A: Characterization of synaptic conductances and integrative properties during electrically induced EEG-activated states in neocortical neurons in vivo. J Neurophysiol 2005, 94(4):2805-2821

6. Jacobson GA, Diba K, Yaron-Jakoubovitch A, Oz Y, Koch C, Segev I, Yarom Y: Subthreshold voltage noise of rat neocortical pyramidal neurones. J Physiol 2005, 564(Pt 1):145-160.

7. Yaron-Jakoubovitch A, Jacobson GA, Koch C, Segev I, Yarom Y: A paradoxical isopotentiality: a spatially uniform noise spectrum in neocortical pyramidal cells. Front Cell Neurosci 2008, 2:3.

8. Bédard C, Destexhe A: A modified cable formalism for modeling neuronal membranes at high frequencies. Biophys J 2008, 94(4):1133-1143.

doi:10.1186/1471-2202-12-S1-P91

Cite this article as: Pettersen et al:: The ball and stick neuron model accounts both for microscopic and macroscopic power laws. BMC Neuroscience 2011 12(Suppl 1):P91.

\footnotetext{
* Correspondence: klas.pettersen@umb.no

Department of Mathematical Sciences and Technology, Norwegian

University of Life Sciences, Ås, 1432, Norway
}

(c) 2011 Pettersen et al; licensee BioMed Central Ltd. This is an open access article distributed under the terms of the Creative 